\title{
An Observational Cohort of First Episode Psychosis in Iran: The Azeri Recent Onset Acute Phase Psychosis Survey (ARAS Cohort) Study Protocol
}

Sara Farhang ( $\sim$ dsfarhang@gmail.com )

University Medical Center Groningen https://orcid.org/0000-0001-8552-138X

Mehrdad Ghaemmaghami

Tabriz University of Medical Sciences

Gholamreza Noorazar

Tabriz University of Medical Sciences

Wim Veling

Academic Medical Centre Groningen University Center of Psychiatry: Universitair Medisch Centrum Groningen Universitair Centrum Psychiatrie

Ayyoub Malek

Tabriz University of Medical Sciences

Alireza Shafiee-kandjani

Tabriz University of Medical Sciences

Mohammad Hossein Somi

Tabriz University of Medical Sciences

Behrooz Z Alizadeh

University Medical Centre Groningen Department of Epidemiology: Universitair Medisch Centrum Groningen Department of Epidemiology

Richard Bruggeman

Rob Giel Research center: Rob Giel Onderzoekcentrum

\section{Study protocol}

Keywords: Schizophrenia, First episode psychosis, Social context, Gene-environment, Cognition

Posted Date: November 5th, 2020

DOI: https://doi.org/10.21203/rs.3.rs-101910/v1

License: (c) (1) This work is licensed under a Creative Commons Attribution 4.0 International License. Read Full License 


\section{Abstract}

\section{Background}

Most of our knowledge about the etiology, course, treatment, and outcome of schizophrenia spectrum and other psychotic disorders stems from Western countries. Data from populations living in other geographical areas and low- and middle-income countries, with different genomes (ethnicity) and exposomes (e.g., culture and social support, drugs of abuse, religion), will add to our knowledge of this complex disorder.

\section{Methods}

The Azeri Acute phase/Recent onset psychosis Survey (ARAS) has been initiated to study the course of the disorder in patients with recent onset psychosis using validated diagnostic tools and a comprehensive outcome monitoring system, aiming to reveal indicators for understanding the risk and resilience factors and for choosing the best personalized treatment strategy. All participants will be evaluated for clinical signs and symptoms as well as risk and resilience factors and will be followed up for 1,3 , and 5 years for outcomes in several domains. A hierarchical cluster method will be applied to identify the number of clusters for each outcome. Defined models will be applied to assess the predictive value of cognition on symptomatic and functional outcomes at follow-up.

\section{Discussion}

The ARAS cohort will yield significant academic- (research and education) and care-related achievements. ARAS data and experience will have value both in being a useful model for other parts of this region and in expansion of the current available knowledge.

\section{Background}

Schizophrenia, as the prototype of schizophrenia spectrum (SSD) and other psychotic disorders, (1) remains one of the most costly disorders in terms of human suffering and societal expenditure. $(2,3)$ It contributed $1.7 \%$ of total years of life lived with disability (YLDs) to the global burden of disease in 2016. The incidence of schizophrenia varies across and within countries, as a wide distribution range is noticeable in available studies. $(4,5)$ A systematic review of 161 studies reported a median incidence rate of 15.2 per 100,000 , with significant effects of migrant status, gender, and urbanicity. $(6,7)$ There have also been differences noted in point and lifetime prevalence estimates related to economic status and latitude. $(8,9)$ The course of illness may also differ across contexts. Older studies suggested that the course of psychotic disorders may be more favorable in low- and middle-income countries (LMIC) (WHO 10 country study), while other studies point at a large treatment gap as a cause of poor outcome in LMIC. (10) Socio-cultural differences such as social support, religious and cultural believes, and differences in mental health care organization might explain a substantial portion of these epidemiological variations. 
SSDs and other psychotic disorders are characterized by positive symptoms including hallucinations and delusions, negative symptoms such as apathy and social withdrawal, and by cognitive impairment. Somatic comorbidity shows a high prevalence, of which the most common types include metabolic syndrome, diabetes mellitus, and cardiovascular diseases,(12) adding to a remarkable increase in the rate of all-cause mortality in recent decades.(9)

SSDs are considered complex genetic disorders. The heterogeneous clinical presentation most likely results from a variety of gene-environment interactions.(13) A wide body of evidence suggests that highly dynamic multi-dimensional interactions between small effects of the genome and exposome underlie the etiopathogenesis of SSDs. $(14,15)$ The exposome encompasses environmental factors, such as social, physical, chemical, biological, and behavioral patterns, over a lifetime, which can all vary widely between populations, in a similar manner to the genome.(16)

Antipsychotic medication, cognitive-behavioral therapy, and rehabilitation are the cornerstones of treatment. Antipsychotic medications, the underlying illness, and their interaction increase the risk of cardiometabolic abnormalities in patients with SSD.(17-19) Here, lifestyle and diet affect this process and, again, these might differ substantially between populations.

Of note, an incomplete response to pharmacotherapy is seen in 30 to $40 \%$ of patients.(20) This proportion has remained almost unchanged over time, despite several decades of pharmacological improvements and the discovery of new medications.(21) Meanwhile, studies continue to examine indicators for a better response to a specific medication or liability for side-effects.(22) However, the predictive value of this personalized approach still needs to be increased before it can be applied to clinical practice.

Despite the differences in genomes, exposomes, and lifestyles across populations, most of the available knowledge about risk and resilience factors for SSD have been collected in high income Western countries. Recently, some studies from Asia have enriched the current knowledge with regard to the role of diverse ethnicity and different living situations on SSD. $(23,24)$ Beyond ethnicity, factors such as childhood adversity, trauma, use of illicit drugs, neurocognitive abilities, social and familial support, and genetic factors all constitute well established modulators of SSD. So far, there are very limited data on SSD from the Middle East and central Asia, including Iran, and specifically from populations with Turkish heritage. Thus, little is known about the attribution of environmental factors and genetics to the development of SSD in Middle Eastern and central Asian populations. Given differences in genetics, culture, lifestyle, and population beliefs in these middle- and low-income countries, such as Iran, it is expected that risk and resilience factors, the magnitude of their interaction, as well as the course of SSD are different from those of Western, high income countries. Investigating these differences between populations and their roles in the development and course of SSD provides a unique opportunity to understand the full spectrum of factors involved in the etiology and pathogenesis of SSD and other psychotic disorders. 
Diagnostic procedures, treatment, and rehabilitation in Iran are based on well-known international diagnostic classification systems and up-to-date western guidelines.(25) Nevertheless, serious gaps in indepth research with a focus on the causes, management, and consequences of SSD exist in this population. $(10,26,27)$ Looking at Iran as an example of a naturally rich, but low-income country, we previously observed patients with SSD and their families facing a variety of social, familial, and economic challenges. $(28,29)$

Accurate knowledge on the burden of SSD on the personal, societal, and health care systems is lacking, as comprehensive national registry data are not yet available. For the same reason, little is known about the outcome of Iranian patients with SSD. It can be argued that the "real-world" implementation of evidence-based practices will be hampered by social and economic factors resulting in sub-optimal care of Iranian patients. Therefore, it is essential to establish well-designed sufficiently large longitudinal studies to investigate the risk and resilience factors involved in SSD in Middle East regions. We therefore initiated the Azeri Recent onset / Acute phase psychosis Survey (ARAS), which has been initiated to study the course of recent onset psychosis by using validated diagnostic tools, setting up a comprehensive outcome monitoring system, aiming to reveal indicators for understanding the risk and resilience factors associated with SSD, and for choosing the best personalized treatment strategy. Next, to improve mental health care, the ARAS-project will define and validate the first population-specific guideline for the management of SSD in Iran. Participants will be followed up at years 1,3 , and 5 after recruitment by face to face interview using structured validated questionnaires. The specific objectives of this study are to (I) determine the disorder course regarding clinical and social aspects and predefined subtypes, (II) identify genetic and environmental risk and resilience factors, (III) investigate whether SSD subtypes are associated with clinical and social aspects and can predict patient prognosis, and (IV) integrate findings from objectives I to III to formulate patient-centered management of psychosis.

\section{Methods}

\section{Study population}

The catchment area for this observational cohort study is East Azerbaijan, a province in the North of Iran with more than 3,700,000 inhabitants, with the majority having an Azeri ethnic background. Iranian Azeris are the largest minority in the country with a Caucasian ethnic background, and are the world's largest Azeri population who are a Turkic ethnic group. The dominant religion in this region is Islam.

\section{Eligibility criteria}

The study target population includes all inhabitants of East Azerbaijan province who are referred with (signs of) a first psychotic episode or evaluated for a recurrent psychotic episode that was previously undiagnosed ( $<2$ years). The cohort will include patients with a diagnosis of schizophrenia, schizophreniform disorder, delusional disorder, brief psychotic disorder, schizotypal personality disorder, schizoaffective disorder, or substance-induced psychotic disorder based on the Diagnostic and Statistical Manual of Mental Disorders (DSM)-5.(1) 


\section{Study Set-up}

The ARAS project was approved by the ethical committee of the National Institute for Medical research Development (NIMAD) in Iran in 2017 (record number: IR.NIMAD.REC.1396.101). All study procedures will be carried out according to the Declaration of Helsinki. Written informed consent will be obtained from all participants and their caregivers or legal guardians. Patients can leave the study at any time for any reason with no influence on their quality of care. A code will be given to each patient and all data will be recorded anonymously. The participants will be informed that all study findings will be stored and handled in strict confidence according to national guidelines. The principal investigator and the main investigators have access to the materials.

This project started in January 2018. For the first baseline assessment (referred to as T0, Figure 1), clinical interviews and assessments will take place at the treating clinics, starting at the time of admission or as soon as possible if forced admission is required. Psychological testing will be performed within a one-month period after inclusion. Diagnostic interviews, questionnaires, and other instruments will be conducted in Farsi, the official language of the country.

Upcoming assessment as routine outcome monitoring will be scheduled for all patients at the one year follow up (T1), which will include the same assessments used in T0, including outcome measurements and assessment for direct costs. The second follow-up (T3) is scheduled at 3 years and the final followup assessment at 5 years (T5) after inclusion. Treatment strategy and visit intervals will be defined by the treating psychiatrist between inclusion and follow-up periods.

\section{Assessments}

Evaluation of patients will be performed in separate meetings depending on their availability (Figure 1). Clinical assessments will be performed by test psychologists/psychiatrists who are trained by repeated workshops.

\section{Clinical diagnosis and biography}

Clinical diagnoses will be made by referring psychiatrists from in- and out-patient clinics. The clinical diagnosis within the SSD will be confirmed by the Structured Clinical Interview for DSM-5 (SCID),(30) or for patients $<18$ years by the Kiddie-Schedule for Affective Disorders and Schizophrenia Present and Lifetime Versions (K-SADS-PL).(31) Farsi versions of these two questionnaires have been used by several research teams inside Iran and contain few cultural adaptations.(32)

Dimensions of Psychosis will be rated using the symptom severity dimension tool of DSM-5.(33) Severity of symptoms will be rated using the Positive and Negative Syndrome Scale (PANSS), which is a semistructured interview on the symptom severity including three subscales of positive and negative symptoms and general psychopathology.(34) The severity of depressive symptoms will be measured using the Calgary Depression Scale in Schizophrenia (CDSS).(35) This instrument differentiates between depression and the negative and positive symptoms of schizophrenia.(36) 
Side-effects of antipsychotics and the patients' adherence to their medication will be assessed using the Subject's Response to Antipsychotics (SRA-34).(37) Evaluation for hyperkinesia, parkinsonism, akathisia, and dystonia will be performed using the St. Hans Rating Scale (SHRS).(38)

Medical history will be obtained from the patient and family members (mostly parents). The nature, severity, and consequences of symptoms for daily functioning will be recorded, and, besides their medication-history, nicotine, illicit drug and alcohol use, and history of illness in the family will be recorded. The biography focuses on signs of an early developmental disorder, current living situation, occupation and daytime activities, and social interactions.

\section{Medical assessments}

A physical health status examination, including a physical examination and measurement of blood pressure, heart rate, length, weight, and waist circumference will be carried out. Laboratory tests will be conducted to test for general health conditions, including complete blood cell count, lipid profile, fasting blood sugar and $\mathrm{HbA1c}$, liver function, kidney, and pituitary function, and urine screening for drugs (morphine, amphetamine, cannabis, tramadol, methadone if indicated). If necessary, additional tests will be performed based on clinical indications, e.g., brain imaging, electroencephalography, or electrocardiography. The requirement for external consultation for any general medical condition will be decided by the psychiatrist and results will be recorded.

\section{Risk and resilience factors}

The evaluation process will continue with semi-structured interviews and self-report questionnaires in different domains. History of experienced adversities during early life and peer interaction in primary school will be evaluated using the Illinois Bully Scale,(39) Olweus Bully/Victim Questionnaire,(40) and the Retrospective bullying experience.(41) The Illinois Bully Scale includes items that address how often a youth is engaged in social aggression, physical and verbal types of victimization, and physical fighting with peers.(35) The Olweus Bully/Victim Questionnaire includes the initiation of an act of bullying against the participant, as well as the expression of bullying behavior against others. The questions include several aspects of bully/victim problems including physical, verbal, indirect, racial, and sexual bullying annoyance. The questionnaire includes pro-bullying and pro-victim attitudes and the reaction of teachers, peers, and parents to the bullying behavior. (36) The Retrospective bullying experience addresses the adult population. It measures the frequency, significance, and extent of bully-victimization. Important aspects like bully-related psychological trauma, suicidal ideation if bullied, and bullying in college and the workplace are also addressed. (37)

Life events will be recorded using the modified Holmes-Rahe stress scale.(42) This inventory includes 50 stressful events adapted to the cultural and social context of the Iranian population that are evaluated by Yes/No questions. The Internalized Stigma of Mental Illness scale (ISMI), a self-report questionnaire measuring self-stigma among persons with psychiatric disorders,(43) will also be used. The 
Multidimensional Scale of Perceived Social Support (44) will evaluate the extent of support from family, friends, or significant people in patients' lives.

Religiosity will be measured using a scale based on Stark and Glock's dimensions of religiosity. This scale is adapted to the Islamic religion measuring dimensions of religious beliefs, practice, experience, and consequences with 26 questions.(45)

Assessment of functioning will include i) WHO Disability Assessment Schedule (WHODAS 2.0),(46) a standard tool applicable to clinical and general populations that covers six dimensions of cognition, mobility, self-care, getting along, life activities, and participation;(42) ii) Global Assessment of Functioning (GAF) Scale form DSM (1) and iii) the three item Functional Recovery Tool including daily living and self-care, work, study, and housekeeping, and social contacts. $(47,48)$ The Manchester Short Assessment of Quality of Life (MANSA) (49) will be used to assess satisfaction with life as a whole and with several domains like leisure, relationships, and mental health.(43)

\section{Cognitive assessments}

A comprehensive neurocognitive battery has been designed in accordance with the recommendations of the Measurement and Treatment Research to Improve Cognition in Schizophrenia (MATRICS) initiative, (50) including eight cognitive domains (see Table 1).

Working memory will be tested with the forward and backward digit span and letter-number sequencing tasks.(51) Divided attention, which is closely tied to executive functioning,(52) will be assessed using the comprehensive (part B) test of the Trail making task. Verbal memory will be tested using The Rey Auditory Verbal Learning Test, measuring memory span, new learning, retention, recognition, and delayed recall.(53) Visual learning will be measured using the Rey-Osterrieth Complex Figure. Executive functioning will be evaluated using the symbol digit modality task, letter digit modality task, backward digit span, and letter-number sequencing task. Speed of information processing will be measured with the average score of both the basic (part A) tests of Stroop (54) and Trail making, (55) measured as time to complete in seconds, as well as category fluency by naming items from three different categories. The Stroop test will also give an estimation about inhibition and flexibility. Intelligence will be tested using the Wechsler Intelligence Scale IV short version,(56) validated for the Iranian population. Regarding social cognition, emotional recognition will be evaluated using the Reading the mind in the eyes test.(57) The Benton Facial Recognition Test will be used to evaluate performance in face discrimination. Theory of mind will be tested using a Sally-Ann story in the Farsi language.

\section{Blood sampling}

A trained nurse will collect $10 \mathrm{~mL}$ venous blood samples in EDTA (ethylenediaminetetraacetic acid) tubes by means of a standard venipuncture at the local hospital laboratory. An individual specific barcode for each patient will be automatically assigned to each tube. This scannable code is unique for every study subject and does not contain elements of their demographics. The code will identify study subjects 
throughout documentation and evaluation and will be traceable only by the principal investigators. The biobank, located at the study coordinating center, will be monitored by the principal investigator.

Separate samples of whole blood, red blood cells, buffy coat, plasma, and serum will be coded and stored in freezers at $-80^{\circ} \mathrm{C}$ available at the sampling site until further analysis.

\section{Follow-up}

All of the described assessments will be repeated during follow-up visits at 1, 3 and 5 years. Relevant outcomes will be measured on three different domains of i) clinical outcome (i.e., symptoms, treatment efficacy, tolerability and cost, diagnostic stability), ii) cognition, and iii) social functioning and recovery (Table 1).

Patients will be invited and interviewed again to assess diagnostic stability and all symptoms will be recorded. Other assessments will include physical examination and laboratory test mentioned earlier, any change in living situation and functioning of the patient, performance on neuro- and social cognition tests, function and disability, perceived stigma, components of metabolic disturbances, and all-cause mortality. Number of relapses, referral to the mental health care system, and re-hospitalization will be recorded.

\section{Data Analysis}

Primary outcomes will be measured in two categories, symptom remission, including cognitive performance, and functional remission. Analysis will be performed to relate risk/resilience factors to the outcome measures and evaluate the course of outcome. We considered five types of outcomes: (1) dimension of psychosis,(58) (2) cognitive functioning,(58) (3) symptom remission,(59) (4) diagnostic stability,(60) and (5) functional remission.(61) Based on rates reported by previous studies, a minimum number was calculated to be 374 patients. Allowing for dropouts during follow-up, we estimated the total sample size to be 500 .

At the level of measurement, inter-rater concordance will be examined for observer rated tools. Raw data will be checked for invalid or nonsense entries. Some raw data will be recoded to composite measures and data for investigators. For example, each component of the cognitive battery might have several tasks and repetitions. These multiple results will be combined to give a summary score based on the structure of the battery. A similar process is also applicable to the other measures.

Descriptive statistics using tabulations and graphical methods will be performed to achieve objective I, after double-checking data entry, data cleaning, and checking for consistency between different types of measurements. Neuropsychological test scores will be converted to domain z scores for correlational analysis to determine predictors of decline or reserve. For other questionnaires, there are predefined values for interpretation. After identifying the distribution of the data, kappa coefficients, correlations, multilevel regression, multidimensional analysis, cluster analysis, and longitudinal trajectory analysis will be used. 
The magnitude of the difference between risk and resilience factors will be evaluated by comparing standardized mean differences to achieve objective II. The relationship between risk and resilience factors will be assessed by logistic regression (for binary outcomes) and multivariable linear regressions (for continuous normally distributed outcomes). Appropriate longitudinal data methods will be used for the assessment of disease progression from baseline first episode status to disease at follow-up in years 1,3 , and 5 . Multivariable models will determine risk factors that predict cognitive, personal, and clinical outcomes, when appropriate. Multilevel analysis to compare disease progression will also be considered.

We will perform a cluster analysis to achieve objective III. The hierarchical cluster method will be applied to identify the number of clusters for each outcome and seed points for a k-means cluster analysis. As an example, neurocognitive subtypes will be compared on baseline clinical and socio-demographic variables by conducting Chi-square analyses or Kruskal Wallis tests on gender, age, social support, educational level, substance use, schizophrenia spectrum disorder diagnosis, symptom severity, dosage of antipsychotic medication, and adverse life events.

We will define predictive models for objective IV to assess the predictive value of (using the previous example) cognition on symptomatic and functional outcomes at follow-up. Separate clinically and statistically meaningful trajectories will be identified using censored normal group-based trajectory modeling (62) and evaluated for model classification accuracy. Predicted trajectories will be evaluated using a random-effects ordinal regression analysis.

\section{Discussion}

The ARAS cohort study will have additive value by characterizing SSD and other psychotic disorders in a population living in the Middle East. Given the extended differences between various aspects of life in Middle Eastern and Western countries, it is highly expected that the ARAS will offer novel understanding about disease mechanisms and outcomes in SSD. However, the most important novel aspect is the study population itself. Several differences are hypothetically present when available studies are compared to our population. Iranian patients may not only have a slightly different genetic susceptibility, especially with respect to population-specific rare genetic variants compared to other countries, but they live in quite different environmental conditions with respect to social support, public stressors, religion, culture, eating habits, inequality, lifestyle, and other risk/resilience factors that may influence the course of the disorder. This diversity provides valuable and rich untouched research material. A first example is illicit drugs,(63) as there is an obvious difference between Iran, with opioid as the most common drug, and European countries, where cannabis use is more prevalent. Another example would be different lifestyles and dietintakes that putatively influence the emergence of metabolic disturbances in patients with psychotic disorders.(64) The ARAS study is also the first study on quality of care for patients with SSD in Iran. Gathering information on a wide variety of treatments, the project per se will improve the quality of care for participating patients and can stand as the first step in this pathway. 
The project might face a number of limitations that come with longitudinal natural cohort studies. Data collection may be hampered by the duration of the diagnostic battery and arrangements. Training of the interviewers and use of standardized procedures for data collection is expected to contribute to a low proportion of missing data, and no imputation is being planned. Patients may be lost to follow-up because of e.g., distance, stigma, and lack of insight. Incomplete data from illiterate patients is another challenge; however, illiteracy will not interfere with many aspects of evaluations, such as the diagnostic interview and several parts of the cognitive battery as well expected outcome. On the other hand, improvement in the care provided during the project might increase cooperation of patients and their families.

The ARAS cohort will yield significant academic- (research and education) and care-related achievements. This study will provide novel data about risk and resilience factors for SSD. The National health care system will benefit from the reliable data on care for patients with SSD. ARAS data and experience will have value both in being a useful model for other parts of this region and in expansion of the current available knowledge. Eventually, ARAS will help to optimize healthcare for people with SSD and improve quality of life of this vulnerable group of people.

\section{Declarations}

- Ethics approval and consent to participate

ARAS project is approved by the ethical committee of National Institute for Medical research Development (NIMAD) in Iran in 2017 (record number: IR.NIMAD.REC.1396.101).

- Consent for publication

Not applicable.

- Availability of data and materials

Data sharing is not applicable to this article as no datasets were generated or analyzed during the current phase.

- Competing interests

The authors declare that they have no competing interests.

- Funding

This study is funded by National Institute for Medical research Development (NIMAD), Iran. the funding body has no role in the design of the study and writing the manuscript.

- Authors' contributions 
SF drafted the manuscript and it was modified by all authors. RB, BZA and SF conceived the study and were major contributors to writing the manuscript, all other authors, WV, MG, GM, AM, MHS and AS contributed to the design and study protocol. All authors read and approved the final manuscript.

- Acknowledgements

We are grateful to the colleagues at Tabriz university of medical sciences for supporting this study.

\section{References}

1. American Psychiatric Association. Diagnostic and statistical manual of mental disorders (DSM-5®). American Psychiatric Pub; 2013.

2. Millier $A$, Schmidt $U$, Angermeyer MC, Chauhan $D$, Murthy $V$, Toumi $M$, et al. Humanistic burden in schizophrenia: a literature review. J Psychiatr Res. 2014;54:85-93.

3. Wu EQ, Birnbaum HG, Shi L, Ball DE, Kessler RC, Moulis M, et al. The economic burden of schizophrenia in the United States in 2002. J Clin Psychiatry. 2005;66(9):1122-9.

4. Schofield P, Das-Munshi J, Becares L, Agerbo E. Neighbourhood ethnic density and incidence of psychosis-First and second generation migrants compared. European Psychiatry. 2017;41:S249.

5. Muli A, Sideli L, Capuccio V, Fearon P, Ferraro L, Kirkbride JB, et al. Low incidence of psychosis in Italy: confirmation from the first epidemiological study in Sicily. Soc Psychiatry Psychiatr Epidemiol. 2017;52(2):155-62.

6. McGrath J, Saha S, Welham J, El Saadi O, MacCauley C, Chant D. A systematic review of the incidence of schizophrenia: the distribution of rates and the influence of sex, urbanicity, migrant status and methodology. BMC Medicine. 2004;2(1):13.

7. Kirkbride JB, Fearon P, Morgan C, Dazzan P, Morgan K, Tarrant J, et al. Heterogeneity in incidence rates of schizophrenia and other psychotic syndromes: findings from the 3-center AeSOP study. Arch Gen Psychiatry. 2006;63(3):250-8.

8. Perala J, Suvisaari J, Saarni SI, et a. Lifetime prevalence of psychotic and bipolar i disorders in a general population. Archives of General Psychiatry pages $=\{19-28\}, .2007 ; 64(1)$.

9. McGrath J, Saha S, Chant D, Welham J. Schizophrenia: a concise overview of incidence, prevalence, and mortality. Epidemiol Rev. 2008;30(1):67-76.

10. Sharifi V, Kermani-Ranjbar T, Amini H, Alaghband-rad J, Salesian N, Seddigh A. Duration of untreated psychosis and pathways to care in patients with first-episode psychosis in Iran. Early Intervention in Psychiatry. 2009;3(2):131-6.

11. Susser E, Martinez-Alis G. Putting Psychosis Into Sociocultural Context: An International Study in 17 Locations. JAMA psychiatry. 2018;75(1):9-10.

12. Islam MA, Khan MH, Quee PJ, Snieder $H$, van den Heuvel, E R, Bruggeman $R$, et al. Familial liability to psychosis is a risk factor for multimorbidity in people with psychotic disorders and their unaffected siblings. European Psychiatry. 2017;45:81-9. 
13. Bernardo M, Bioque M, Cabrera B, Lobo A, González-Pinto A, Pina L, et al. Modelling geneenvironment interaction in first episodes of psychosis. Schizophr Res. 2017;189:181-9.

14. Bernardo M, Bioque M, Cabrera B, Lobo A, González-Pinto A, Pina L, et al. Modelling geneenvironment interaction in first episodes of psychosis. Schizophrenia Research. 2017;189:181-9.

15. Zwicker A, Denovan-Wright EM, Uher R. Gene-environment interplay in the etiology of psychosis. Psychol Med. 2018:1-12.

16. Pekkanen J, Pearce N. Environmental epidemiology: challenges and opportunities. Environ Health Perspect. 2001;109(1):1.

17. Holt RIG, Peveler RC, Byrne CD. Schizophrenia, the metabolic syndrome and diabetes. Diabetic Med. 2004;21(6):515-23.

18. Rady MY, Johnson DJ, Patel BM, Larson JS, Helmers RA. Influence of Individual Characteristics on Outcome of Glycemic Control in Intensive Care Unit Patients With or Without Diabetes Mellitus. Mayo Clinic Proceedings. 2005;80(12):1558-67.

19. Fadai F, Mousavi B, Ashtari Z, Farhang S, Hashempour S, Shahhamzei N, et al. Saffron aqueous extract prevents metabolic syndrome in patients with schizophrenia on olanzapine treatment: a randomized triple blind placebo controlled study. Pharmacopsychiatry. 2014;47(04/05):156-61.

20. Lieberman JA, Tollefson G, Tohen M, Green Al, Gur RE, Kahn R, et al. Comparative efficacy and safety of atypical and conventional antipsychotic drugs in first-episode psychosis: a randomized, doubleblind trial of olanzapine versus haloperidol. Am J Psychiatry. 2003;160(8):1396-404.

21. Menezes NM, Arenovich T, Zipursky RB. A systematic review of longitudinal outcome studies of firstepisode psychosis. Psychol Med. 2006;36(10):1349-62.

22. Hwang R, Zai C, Tiwari A, Muller DJ, Arranz MJ, Morris AG, et al. Effect of dopamine D3 receptor gene polymorphisms and clozapine treatment response: exploratory analysis of nine polymorphisms and meta-analysis of the Ser9Gly variant. The pharmacogenomics journal. 2010;10(3):200.

23. An SK, Kang JI, Park JY, Kim KR, Lee SY, Lee E. Attribution bias in ultra-high risk for psychosis and first-episode schizophrenia. Schizophr Res. 2010;118(1):54-61.

24. Yamazawa R, Nemoto T, Kobayashi H, Chino B, Kashima H, Mizuno M. Association between duration of untreated psychosis, premorbid functioning, and cognitive performance and the outcome of firstepisode schizophrenia in Japanese patients: prospective study. Australian \& New Zealand Journal of Psychiatry. 2008;42(2):159-65.

25. World Health Organization, World Organization of National Colleges, Academies, Academic Associations of General Practitioners/Family Physicians. Integrating mental health into primary care: a global perspective. World Health Organization; 2008.

26. Rezaei O, Khodaie-Ardakani M, Mandegar MH, Dogmehchi E, Goodarzynejad H. Prevalence of metabolic syndrome among an Iranian cohort of inpatients with schizophrenia. The International Journal of Psychiatry in Medicine. 2009;39(4):451-62.

27. Sadeghieh Ahari S, Nikpou H, Molavi P, Abdi R, Amani F, Shirinzadeh B. An investigation of duration of untreated psychosis and the affecting factors. J Psychiatr Ment Health Nurs. 2014;21(1):87-92. 
28. Goreishizadeh MA, Mohagheghi A, Farhang S, Alizadeh L. Psychosocial disabilities in patients with schizophrenia. Iranian journal of public health. 2012;41(5):116.

29. Shamsaei F, Cheraghi F, Bashirian S. Burden on Family Caregivers Caring for Patients with Schizophrenia. Iranian Journal of Psychiatry. 2015;10(4):239-45.

30. Shooshtari MH, Davari-Ashtiani R, Shahrivar Z, Shabani A, Semnani Y, Kaviani H, et al. Structured clinical interview for DSM-IV (SCID Persian translation and cultural adaptation). Iranian journal of psychiatry. 2007;2(1):46-8.

31. Ghanizadeh A, Mohammadi MR, Yazdanshenas A. Psychometric properties of the Farsi translation of the kiddie schedule for affective disorders and schizophrenia-present and lifetime version. BMC Psychiatry. 2006;6(1):10.

32. Yousefi-Nooraie R, Mohammadi MR, Salesian N, Amin-Esmaeeli M, Mansouri N, Mesgarpour B, et al. Prevalence of psychiatric disorders in Iran: A systematic review. Iranian Journal of Psychiatry. 2007;2(4):137-50.

33. Tandon R, Gaebel W, Barch DM, Bustillo J, Gur RE, Heckers S, et al. Definition and description of schizophrenia in the DSM-5. Schizophr Res. 2013;150(1):3-10.

34. Kay SR, Fiszbein A, Opfer LA. The positive and negative syndrome scale (PANSS) for schizophrenia. Schizophr Bull. 1987;13(2):261.

35. Kontaxakis VP, Havaki-Kontaxaki BJ, Stamouli SS, Margariti MM, Collias CT, Christodoulou GN. Comparison of four scales measuring depression in schizophrenic inpatients. European Psychiatry. 2000;15(4):274-7.

36. Addington D, Addington J, Maticka-Tyndale E. Assessing depression in schizophrenia: the Calgary Depression Scale. The British journal of psychiatry. 1993.

37. A Wolters H, Knegtering R, Wiersma D, J van dB. Evaluation of the Subjects' Response to Antipsychotics Questionnaire. ; 2006.

38. Gerlach J, Korsgaard S, Clemmesen P, Lauersen AL, Magelund G, Noring U, et al. The St. Hans Rating Scale for extrapyramidal syndromes: reliability and validity. Acta Psychiatr Scand. 1993;87(4):24452.

39. Akbari Balootbangan A, Talepasand S. Validation of the Illinois bullying scale in primary school students of Semnan, Iran. Journal of Fundamentals of Mental Health. 2015;17(4):11-2.

40. Rezapour M, Soori H, Khodakarim S. Testing Psychometric Properties of the Perpetration of Bullying and Victimization Scales with Olweus Bullying Questionnaire in Middle Schools. Journal of Safety Promotion and Injury Prevention. 2014;1(4):212-21.

41. Schäfer M, Korn S, Smith PK, Hunter SC, Mora-Merchán JA, Singer MM, et al. Lonely in the crowd: Recollections of bullying. British Journal of Developmental Psychology. 2004;22(3):379-94.

42. Malek A, Poorafkary N, Dadashzadeh $\mathrm{H}$, Safaeeyan A. Ranking of stressful life events in general population of Tabriz, Iran. Research Journal of Biological Sciences. 2008;3(9):993-8. 
43. Ritsher JB, Otilingam PG, Grajales M. Internalized stigma of mental illness: psychometric properties of a new measure. Psychiatry Res. 2003;121(1):31-49.

44. Zimet GD, Dahlem NW, Zimet SG, Farley GK. The multidimensional scale of perceived social support. J Pers Assess. 1988;52(1):30-41.

45. Khodayarifard M. Religiosity measuring model and preparation of religiosity scale to Iranian population. ; 2010.

46. Ustun TB. Measuring health and disability: Manual for WHO disability assessment schedule WHODAS 2.0. World Health Organization; 2010.

47. Wiersma D, Visser E, Bähler M, Bruggeman R, Delespaul PA, Keet IP, et al. Functional remission of people with serious mental illness (SMI): psychometric properties of a new ROM-instrument. Tijdschrift voor psychiatrie. 2015;57(6):395-404.

48. Swildens WE, Visser E, Bähler M, Bruggeman R, Delespaul P, van der Gaag M, et al. Functional recovery of individuals with serious mental illnesses: Development and testing of a new short instrument for routine outcome monitoring. Psychiatr Rehabil J. 2018;41(4):341.

49. Bjorkman T, Svensson B. Quality of life in people with severe mental illness. Reliability and validity of the Manchester Short Assessment of Quality of Life (MANSA). Nordic journal of psychiatry. 2005;59(4):302-6.

50. Arranz MJ, De Leon J. Pharmacogenetics and pharmacogenomics of schizophrenia: a review of last decade of research. Mol Psychiatry. 2007;12(8).

51. Crowe SF. Does the letter number sequencing task measure anything more than digit span? Assessment. 2000;7(2):113-7.

52. Salthouse TA. What cognitive abilities are involved in trail-making performance? Intelligence. 2011;39(4):222-32.

53. Rezvanfard M, Ekhtiari H, Noroozian M. The Rey Auditory Verbal Learning Test: alternate forms equivalency and reliability for the Iranian adult population (Persian version). Archives of Iranian medicine. 2011;14(2):104.

54. Stroop JR. Studies of interference in serial verbal reactions. J Exp Psychol. 1935;18(6):643.

55. Spreen O, Strauss E. A compendium of neuropsychologial tests. In: InAdministration, norms, and commentary. Oxford University Press; 1998.

56. Reza JA, Abas P. Reliability and validity of wechsler intelligence scale for childrenthird edition (WISCIII) in Iran. J Med Educ. 2003;2(2):75-80.

57. Khorashad BS, Baron-Cohen S, Roshan GM, Kazemian M, Khazai L, Aghili Z, et al. The "Reading the Mind in the Eyes" test: investigation of psychometric properties and test-retest reliability of the persian version. J Autism Dev Disord. 2015;45(9):2651-66.

58. Albert N, Melau M, Jensen H, Hastrup LH, Hjorthøj C, Nordentoft M. The effect of duration of untreated psychosis and treatment delay on the outcomes of prolonged early intervention in psychotic disorders. NPJ schizophrenia. 2017;3(1):34. 
59. Lally J, Ajnakina O, Stubbs B, Cullinane M, Murphy KC, Gaughran F, et al. Remission and recovery from first-episode psychosis in adults: systematic review and meta-analysis of long-term outcome studies. The British Journal of Psychiatry. 2017;211(6):350-8.

60. Pope MA, Joober R, Malla AK. Diagnostic stability of first-episode psychotic disorders and persistence of comorbid psychiatric disorders over 1 year. The Canadian Journal of Psychiatry. 2013;58(10):588-94.

61. Verma S, Subramaniam M, Abdin E, Poon LY, Chong SA. Symptomatic and functional remission in patients with first-episode psychosis. Acta Psychiatr Scand. 2012;126(4):282-9.

62. Jones BL, Nagin DS, Roeder K. A SAS procedure based on mixture models for estimating developmental trajectories. Sociological methods \& research. 2001;29(3):374-93.

63. Semple DM, Mclntosh AM, Lawrie SM. Cannabis as a risk factor for psychosis: systematic review. Journal of psychopharmacology. 2005;19(2):187-94.

64. Papanastasiou E. The prevalence and mechanisms of metabolic syndrome in schizophrenia: a review. Therapeutic advances in psychopharmacology. 2013;3(1):33-51.

\section{Tables}

Table 1: different domains of data collection at baseline and at 1, 35 years follow-up 


\section{Outcome parameter}

Diagnosis (stability)

Symptom severity

Depressive symptoms

Medication side effect

Metabolic profile

\section{Cognitive outcome}

Working memory

Attention

Visual and auditory memory (immediatedelayed), recognition, retention and learning

Executive functioning

Speed of processing

Intelligence quotation

Social cognition

Theory of mind

\section{Functional outcome}

Disability

Functional remission

Quality of life

Social, occupational, and psychological functioning

\section{Instrument}

Structured psychiatric interview

Positive and Negative Syndrome Scale, Dimensions of Psychosis symptom severity dimension tool of DSM- 5

Calgary Depression Scale in Schizophrenia

St. Hans Rating Scale, Subjective Response to Antipsychotics-34

Laboratory testing, anthropometrics
Forward digit span task, Backward digit span task, Letter-number sequencing task, stroop task

Trail making task

Auditory verbal learning test, Rey Osterrieth complex figure

Symbol digit modality task, letter digit modality task, Verbal fluency task

Stroop task

Wechsler intelligence scale IV

Benton Facial Recognition Test

Sali Ann
WHO Disability Assessment Schedule

Functional remission tool

Manchester Short Assessment of Quality of Life

Global Assessment of Functioning

\section{Figures}




\begin{tabular}{|c|c|c|c|c|}
\hline & \multicolumn{2}{|c|}{ Enrolment } & \multicolumn{2}{|c|}{ Follow up } \\
\hline & 0 & $T 1$ & T3 & T5 \\
\hline Structured psychiatric interview & $x$ & $x$ & $x$ & $x$ \\
\hline Physical examination & $\mathrm{x}$ & & & \\
\hline laboratory tests & $x$ & & $x$ & $x$ \\
\hline Informed consent & $x$ & & & \\
\hline Blood sampling & & $x$ & & \\
\hline Assessments: & & & & \\
\hline Risk and resilient factors & & $x$ & & \\
\hline Clinical symptoms & & $x$ & $x$ & $x$ \\
\hline Level of functioning & & $x$ & $x$ & $x$ \\
\hline Cognitive performance & & $x$ & $x$ & $x$ \\
\hline Medication side effect & & & $x$ & $x$ \\
\hline Received care & & & $x$ & $x$ \\
\hline
\end{tabular}

Figure 1

The schedule of enrolment, assessments and follow up in ARAS study. 